\title{
Excessive Activation of Poly(ADP-Ribose) Polymerase Contributes to Inherited Photoreceptor Degeneration in the Retinal Degeneration 1 Mouse
}

\author{
François Paquet-Durand, ${ }^{1 *}$ José Silva, ${ }^{1 *}$ Tanuja Talukdar, ${ }^{1}$ Leif E. Johnson, ${ }^{1}$ Seifollah Azadi, ${ }^{1}$ Theo van Veen, ${ }^{1}$ \\ Marius Ueffing, ${ }^{2,3}$ Stefanie M. Hauck, ${ }^{2}$ and Per A. R. Ekström ${ }^{1}$ \\ ${ }^{1}$ Department of Ophthalmology, Lund University, 22185 Lund, Sweden, ${ }^{2}$ Institute of Human Genetics, Gesellschaft für Strahlung und Umweltforschung- \\ National Research Centre for Environment and Health, 85764 Neuherberg, Germany, and ${ }^{3}$ Institute of Human Genetics, Technical University of Munich, \\ 81675 Munich, Germany
}

\begin{abstract}
Retinitis pigmentosa (RP) is an inherited blinding disease for which there is no treatment available. It is characterized by a progressive and neurodegenerative loss of photoreceptors but the underlying mechanisms are poorly understood. Excessive activation of the enzyme poly(ADP-ribose) polymerase (PARP) has recently been shown to be involved in several neuropathologies. To investigate the possible role of PARP in retinal photoreceptor degeneration, we used the retinal degeneration 1 (rd1) mouse RP model to study PARP expression, PARP activity, and to test the effects of PARP inhibition on photoreceptor viability. PARP expression was found to be equal between rd1 and wild-type counterpart retinas. In contrast to this, a dramatic increase in both PARP activity per se and PARP product formation was detected by in situ assays in rd 1 photoreceptors actively undergoing cell death. Furthermore, PARP activity colabeled with oxidatively damaged DNA and nuclear translocation of AIF (apoptosis-inducing factor), suggesting activation of PARP as a bridge between these events in the degenerating photoreceptors. The PARP-specific inhibitor PJ34 [ $N$-(6-0xo-5,6-dihydrophenanthridin-2-yl)- $N, N$ dimethylacetamide $\mathrm{HCl}$ ] reduced the number of cells exhibiting death markers in a short-term retinal culture paradigm, a protective effect that was translated into an increased number of surviving photoreceptors when the inhibitor was used in a long-term culture setting. Our results thus demonstrate an involvement of PARP activity in $\mathrm{rd} 1$ photoreceptor cell death, which could have a bearing on the understanding of neurodegenerations as such. The findings also suggest that the therapeutical possibilities of PARP inhibition should include retinal diseases like RP.
\end{abstract}

Key words: blindness; neuroprotection; apoptosis; necrosis; poly(ADP)-ribosylation; neuropathology

\section{Introduction}

Retinitis pigmentosa (RP) is a group of inherited neurodegenerative diseases that attack the retinal photoreceptors. With its high prevalence, $\sim 1: 4000, \mathrm{RP}$ is regarded as the leading cause of blindness and visual disability among younger people in the developed world (Herse, 2005). Over 160 different mutations, encompassing genes coding for proteins with remarkably diverse functions, are known to cause RP (www.sph.uth.tmc.edu/retnet). Surprisingly, however, the pathological mechanisms behind the degen-

\footnotetext{
Received April 4, 2007; revised Aug. 9, 2007; accepted Aug. 11, 2007.

This work was supported by grants from the European Union (RETNET: MRTN-CT-2003-504003; EVI-GENORET LSHG-CT-2005-512036), Swedish Medical Research Council, Foundation Fighting Blindness, Petrus and Augusta Hedlunds Foundation, Dutch Retina Foundation, Kronprinsessan Margaretas Arbetsnämnd för Synskadade, The Crafoord Foundation, The Segerfalk Foundation, K\&E Olssons Foundation, Kungliga Fysiografiska Sällskapet i Lund and Lund University Hospital Funds. We thank Hodan Abdalle and Birgitta Klefbohm for technical assistance and Julianne McCall for helpful comments on this manuscript.

*F.P.-D. and J.S. contributed equally to this work.

Correspondence should be addressed to Dr. François Paquet-Durand, Department of Ophthalmology, University of Lund, Biomedical Center B13, SE-221 84 Lund, Sweden. E-mail: francois.paquet-durand@med.lu.se.

S. Azadi's present address: Biochemistry and Molecular Biology Department, Life Science Institute, University of British Columbia, Vancouver, British Columbia, Canada V6T1Z4

D0I:10.1523/JNEUROSCI.1514-07.2007

Copyright $\odot 2007$ Society for Neuroscience $\quad$ 0270-6474/07/2710311-09\$15.00/0
}

eration have not been resolved for any of these mutations and effective RP treatment is lacking (Delyfer et al., 2004).

The retinal degeneration 1 ( $\mathrm{rd} 1$ ) mouse is a well studied RP model and carries a loss-of-function mutation in the gene for the $\beta$-subunit of the rod photoreceptor cGMP phosphodiesterase 6 (PDE6- $\beta$ ) (Bowes et al., 1990). Similar mutations have been linked to $4-5 \%$ of the RP cases (McLaughlin et al., 1995). PDE6 regulates rod cGMP levels, which in turn govern their cGMPgated cation channels (Cobbs and Pugh, 1985). The nonfunctional PDE6 therefore results in cGMP accumulation and subsequent abnormally high $\mathrm{Ca}^{2+}$ levels in the $\mathrm{rd} 1$ photoreceptors (Farber and Lolley, 1974; Fox et al., 1999). By mostly unknown routes, this leads to an apoptotic-like rod cell death (Chang et al., 1993; Portera-Cailliau et al., 1994), followed by a mutationindependent cone death. Although apoptosis often involves caspases, these play only a minor role, if any, in the rd 1 degeneration. For instance, relevant caspase activation is absent during the degeneration (Kim et al., 2002; Doonan et al., 2003) and interventions of the caspase cascade fail to preserve the rd1 photoreceptors (Yoshizawa et al., 2002; Zeiss et al., 2004).

The poly(ADP-ribose) polymerase (PARP) enzyme is often seen as the "guardian of the genome," because it promotes DNA 
repair whenever the DNA is compromised (Schreiber et al., 2006) and thus continuously protects the cell. However, cell death evoked by exaggerated PARP activity independent of caspases is an emerging concept in neurodegeneration and a number of other severe diseases (Scott et al., 2004; Koh et al., 2005; Jagtap and Szabo, 2005). As a consequence, this has raised hopes for novel therapies based on PARP inhibition.

The neuroretina of the eye represents a readily accessible part of the brain and provides excellent possibilities for investigating degenerative events in the CNS. So far, studies on PARP in the retina have been restricted to model systems with acutely induced retinal cell death (Chiang and Lam, 2000; Goebel and Winkler, 2006; Miki et al., 2007). A possible role for PARP in models of inherited degenerative diseases therefore merits investigation. To this end, we combined here in situ activity analysis, which is a novel approach, with studies of PARP expression and PARP product formation in degenerating $\mathrm{rd} 1$ or healthy wild-type retinas. We furthermore used an in vitro retinal explant system to analyze the effects of PARP inhibition on $\mathrm{rd} 1$ photoreceptor survival. Our results show an unequivocal and significant involvement of PARP activity in the rd1 degeneration.

\section{Materials and Methods}

Animals and tissues. All animals were treated in accordance with the Swedish National Animal Care and Ethics Committee (approval no. M225-04). Congenic wild-type (wt) control mice of the $\mathrm{C} 3 \mathrm{H}$ strain and homozygous rd1 were used for the studies. Day of birth was considered as postnatal day $0(\mathrm{P} 0)$. Handling of animals and tissue has been described in detail previously (Miranda-Sanz et al., 2007).

Transcription assay. A transcription assay, described in detail by Azadi et al. (2006), was performed on a custom-made mouse gene chip. Two times upregulation or downregulation in at least three of five hybridizations was defined as the significance level of variation. Results not pertaining to the present study have been published previously.

Immunostaining. Immunostaining of cryosectioned retinas of either in vivo or explant origin was performed as described previously (Azadi et al., 2006, 2007). For dilution of primary antibodies, see Table 1. Controls were performed on both wt and rd1 tissue and consisted of omitting the primary antibodies with application of the fluorescence detection system only. For full views of negative controls, please refer to supplemental Figure 1 (available at www.jneurosci.org as supplemental material).

PARP activity assay in situ. For the PARP enzyme activity assays, we adopted a technique originally presented by Bakondi et al. (2002). In brief, eyes were collected from both $\mathrm{rd} 1$ and wt mice and immediately frozen on dry ice $\left(-72^{\circ} \mathrm{C}\right)$. The whole eyes were embedded in TissueTek (Sakura Finetek, Zoeterwoude, The Netherlands) and cryosectioned at $12 \mu \mathrm{m}$. A biotin-avidin blocking kit (Vector, Burlingame, CA) was used to block endogenous biotin and to reduce background. PARP reaction mixture $\left[10 \mathrm{mM} \mathrm{MgCl}_{2}, 1 \mathrm{~mm}\right.$ dithiothreitol, $5 \mu \mathrm{M}$ biotinylated NAD (Trevigen, Gaithersburg, MD) in $100 \mathrm{~mm}$ Tris buffer with $0.2 \%$ Triton $\mathrm{X}-100, \mathrm{pH} \mathrm{8.0]}$ was applied to the sections for $2 \mathrm{~h}$ at $37^{\circ} \mathrm{C}$. After three 5 min washes in PBS, incorporated biotin was detected by fluorescently labeled avidin (1:800 in PBS, $1 \mathrm{~h}$ at room temperature). After three $5 \mathrm{~min}$ washes in PBS, the sections were mounted in Vectashield (Vector). Controls included use of reaction mixture containing structurally different PARP inhibitors, either $6 \mu \mathrm{M} \mathrm{N}$-(6-oxo-5,6-dihydrophenanthridin-2yl)- $N, N$-dimethylacetamide $\cdot \mathrm{HCl}$ (PJ34), $80 \mu \mathrm{M}$ 3,4-dihydro-5-[4-(1piperidinyl)butoxy]-1(2H)-isoquinolinone (DPQ), or $730 \mu \mathrm{M} 3$-aminobenzamide (3-ABA) (all from Merck, Darmstadt, Germany), which in all cases prevented formation of detectable reaction product (data not shown). Likewise, omission of the biotinylated NAD from the reaction mixture resulted in absence of detectable reaction product on both wt and $\mathrm{rd} 1$ retinal sections (see Fig. $2 B$ ).
For the colocalization of PARP activity with poly(ADP-ribose) (PAR) immunofluorescence, the activity stained sections were postfixed in $4 \%$ paraformaldehyde (PFA), washed four times for $5 \mathrm{~min}$, and then stained for PAR immunoreactivity as above, with a corresponding secondary antibody.

Terminal deoxynucleotidyl transferase-mediated biotinylated UTP nick end labeling assay. For the visualization of dying cells (Gavrieli et al., 1992), a terminal deoxynucleotidyl transferase-mediated biotinylated UTP nick end labeling (TUNEL) assay kit conjugated with TMR red from Roche Diagnostics (Mannheim, Germany) was used on retinal sections and performed as stated in the instructions. Controls consisted of omitting the terminal deoxynucleotidyl transferase enzyme from the labeling solution (negative control) and in pretreating the sections for $30 \mathrm{~min}$ with DNase I (Roche Diagnostics; $3 \mathrm{U} / \mathrm{ml}$ ) in $50 \mathrm{~mm}$ Tris-HCl, pH 7.5, 1 $\mathrm{mg} / \mathrm{ml} \mathrm{BSA}$ to induce DNA strand breaks (positive control). Negative control gave no staining at all, whereas positive control stained all nuclei in all layers of the retina (see Fig. 2C).

For the colocalization of PAR and TUNEL, sections were washed four times in PBS after immunofluorescence, incubated with $20 \%$ goat serum for $1 \mathrm{~h}$, and underwent the TUNEL assay.

For the colocalization of PARP activity with TUNEL, the Apoptag assay kit from Chemicon (Temecula, CA) was used. The PARP activity assay on unfixed retinal sections was followed by a fixation step in $4 \%$ PFA for $1 \mathrm{~h}$. Sections were then washed four times in PBS, and then the Apoptag assay was performed as stated in the manual.

Detection of oxidatively damaged DNA. To detect oxidatively damaged DNA in rd1 photoreceptors, retinal sections were incubated with fluorescently labeled avidin (Invitrogen, Carlsbad, CA) for $1 \mathrm{~h}$ at a dilution of 1:80 in PBS (Miranda-Sanz et al., 2007). Negative controls (see Fig. 3N) were treated with avidin staining solution preadsorbed $(1 \mathrm{~h}$ at room temperature) with 12 nм 8-hydroxy-2'-deoxyguanosine (Calbiochem, La Jolla, CA) and 50 nm biotin (Vector). Because the PARP activity assay also uses avidin binding, it could not be combined with avidin labeling of damaged DNA. To colabel for PARP activity, we stained instead for PAR, which was performed as above followed by $1 \mathrm{~h}$ incubation with labeled avidin.

Cell counting procedures. To assess the number of TUNEL, PAR immunofluorescence, or PARP activity positive cells, pictures were taken from retinal sections at $40 \times$ magnification, and the number of positive cells was counted manually. The total number of cells was determined by measuring the outer nuclear layer (ONL) area with the aid of Zeiss (Jena, Germany) Axiovision 4.2 software and dividing it through the average cell size. The number of positive cells was then divided by the total number of cells in the ONL to give the percentage of positive cells. For each animal, three sections were quantified and averaged. Between three and eight different animals were analyzed for each time point, assay type, and genotype.

The number of photoreceptor rows in the ONL after long-term culture was determined by counts on 36 sections taken from four different and separated cutting levels within a retinal explant. For each count, a vertical column was placed on six to seven different positions of the section, and the photoreceptor nuclei in each of these positions were counted manually. These counts were averaged to give the mean value of photoreceptor rows for one explant. For each experimental group, six explants from six different animals were counted in this way.

Western blotting. Retinas from $\mathrm{P} 11$ animals were removed in dissecting buffer (10 mM Tris, 1 mM EDTA, $150 \mathrm{~mm} \mathrm{NaCl}, 1 \mathrm{~mm} \mathrm{Na}_{3} \mathrm{VO}_{4}, 50 \mathrm{~nm}$ 
okadaic acid, $\mathrm{pH}$ 7.3) supplemented with protease inhibitor mixture (Roche), pooling both retinas from one animal for each sample and making three samples for each genotype. The tissue was homogenized in sample buffer [for PARP: $2 \%$ SDS, $10 \%$ glycerol, 0.0625 м Tris- $\mathrm{HCl}, \mathrm{pH}$ 6.8; for PAR: 4\% CHAPS (3-[(3-cholamidopropyl)dimethylammonio]1-propanesulfonate), $9 \mathrm{M}$ urea, $\mathrm{pH} 7.2$ ] first by mechanical force and then using ultrasound. The sample was centrifuged for $10 \mathrm{~min}$ at $14,000 \times \mathrm{g}$, and the supernatant was then removed and stored at $-80^{\circ} \mathrm{C}$. Bio-Rad (Hercules, CA) DC Protein Assay kit was used to determine protein concentrations, and either 5,10 , or $20 \mu \mathrm{g}$ of protein from each sample was separated by SDS-PAGE on 6,12 , or $4-12 \%$ gradient gels, which were then transferred onto Immobilon-P polyvinylidene difluoride membranes (Millipore, Billerica, MA). Membranes were incubated for at least $1 \mathrm{~h}$ in blocking buffer (5\% dried milk in PBS with $0.1 \%$ Tween 20 ) and then kept overnight at $4^{\circ} \mathrm{C}$ with antibodies against PARP- 1 or PAR in blocking buffer (1:5000 or 1:1000, respectively). The day after, the membranes were washed, treated for $1 \mathrm{~h}$ with horseradish peroxidaseconjugated secondary antibodies, and visualized using the ECL Plus Western Blotting Detection system (Amersham Biosciences, Sunnyvale, $\mathrm{CA}$ ) and Hyperfilm (Amersham Biosciences). Films were digitized using a GS-710 Calibrated Imaging Densitometer (Bio-Rad), and signal intensities were quantified with QuantityOne (version 4.2.1; Bio-Rad).

PARP inhibitor treatment of retinal cultures. To study the effect of pharmacological PARP inhibition on the rd1 photoreceptor degeneration, retinal explant cultures were used. Retinal explants were prepared, with small exceptions, as described previously (Ahuja et al., 2005; Miranda-Sanz et al., 2007). Animals of P5 age were used for retinal explant cultures including the retinal pigment epithelium. Retinas were cultured in serum free R16 medium (Invitrogen) on culture dish inserts (Millipore) and placed into six-well culture plates (Nunc, Roskilde, Denmark). Culture plates were kept in an incubator with $100 \%$ humidity and $5 \% \mathrm{CO}_{2}$ in air at $37^{\circ} \mathrm{C}$. Medium was exchanged every second day.

Retinal explants were allowed to adjust to culture conditions for $2 \mathrm{~d}$ without treatment and then treated with PJ34 at a concentration of $6 \mu \mathrm{M}$ in $1 \%$ DMSO, whereas controls received 1\% DMSO only. PARP inhibitor treatment lasted for either $4 \mathrm{~d}$ (short-term culture) or $18 \mathrm{~d}$ (long-term culture) to ages corresponding to P11 or P25, respectively. After completion of the culture period, the explants were embedded, sectioned, and stained as mentioned above. To study the morphology and for cell counting, cryosections were stained with hematoxylin/eosin.

Microscopy and statistics. Morphological observations and routine light microscopy were performed on a Zeiss Axiophot microscope equipped with a Zeiss Axiocam digital camera. Images were captured using Zeiss Axiovision 4.2 software; image overlays and contrast enhancement were done using Adobe Photoshop 6.0. Images are representative for stainings from at least three different animals from each genotype. Stainings depicted in one column were processed under identical conditions. Statistical comparisons among different experimental groups were made using a two-tailed Student's $t$ test and Microsoft Excel software. Error bars indicate SEM; scale bar is $50 \mu \mathrm{m}$.

\section{Results}

\section{PARP expression in $\mathrm{rd} 1$ and wt retina}

Total PARP expression was studied in $\mathrm{rd} 1$ and wt mice at P11. P11 animals were used, because at this stage the rd1 ONL shows abundant photoreceptor cell death, whereas its thickness is still comparable with that of wt animals (Hauck et al., 2006; Azadi et al., 2007). The use of such samples thus reduces any possible bias in gene expression patterns caused by unproportional contribution of certain retinal layers. At the same time, the high incidence of rd1 photoreceptor degeneration at P11 permits detection of differentially regulated genes and proteins.

Previously, we used microarray techniques to demonstrate differential gene regulation between $\mathrm{rd} 1$ and wt retina at $\mathrm{P} 11$ (Azadi et al., 2006). Using data generated at that time, but not presented before, we here report that the transcription levels of all PARP genes included on the array (PARP-1, -2, -6, -7, -8, -14,
-16) were unchanged between wt and $\mathrm{rd} 1$ mouse retinas (ratio $\mathrm{rd} 1 / \mathrm{wt} \cong 1$ ) (Fig. $1 A$ ). PARP activity is counterbalanced by the activity of poly(ADP-ribose) glycohydrolase (PARG), which rapidly degrades the PAR chains formed by PARP (Bonicalzi et al., 2005). The PARG gene was also included on the microarray, but again no difference in gene transcription was detected (Fig. $1 A$ ).

PARP-1 is the best known variant of the PARP isoforms and relevant for considerations on pathophysiology and experimental therapy (Jagtap and Szabo, 2005). An antibody against PARP-1 (hereafter only PARP) was used for immunofluorescent staining of P11 rd1 and wt retinas, which showed that PARP was present preferentially in the nuclei of most, if not all, retinal cells but without obvious qualitative or quantitative differences between the two genotypes (Fig. $1 B, C$ ). To quantify PARP protein expression levels, the same antibody was used in Western blotting of retinal tissues from P11 (Fig. $1 D$ ), where it revealed a $116 \mathrm{kDa}$ band that corresponds to the molecular weight of PARP. There were no quantifiable differences between $\mathrm{rdl}$ and wt samples, confirming the immunostaining and microarray results.

\section{PARP activity is increased in rd1 photoreceptors}

The above results did not reveal any difference in PARP expression levels between $\mathrm{rd} 1$ and wt retinas. However, this does not exclude the possibility of differential regulation of PARP activity. To address this question, we used two different experimental approaches. First, an antibody directed against PAR, the product of PARP, was used as an indirect marker for PARP activity. In the following, we refer to the catalytic activity of PARP as poly(ADP)ribosylation. Figure $1 E$ shows increased poly(ADP)-ribosylation of proteins in rd1 retinas, as judged by Western blotting of P11 samples. Both wt and $\mathrm{rd} 1 \mathrm{retinal}$ samples contained a poly(ADP)ribosylated protein of $\sim 116 \mathrm{kDa}$, most likely corresponding to PARP, because the latter is known to be capable of autopoly(ADP)-ribosylation (Mendoza-Alvarez and AlvarezGonzalez, 1993). The $116 \mathrm{kDa}$ band was the only reaction observed in wt retinal samples, but in $\mathrm{rd} 1$ samples this band was overshadowed by a group of bands, which stretched between 140 and $240 \mathrm{kDa}$ in a smear-like manner. Interestingly, the latter outcome has a striking resemblance with the poly(ADP)ribosylation profile of injured mouse brain neurons (Fossati et al., 2007).

Furthermore, PAR antibody immunofluorescence on cryosectioned retinas revealed a strong and consistent PAR staining in a subset of cell bodies in P11 rd1 photoreceptors but not in wt counterparts (Fig. 2A,D; for views of full retinal cross sections, refer to supplemental Figure 2, available at www.jneurosci.org as supplemental material). The frequency of PAR-positive $\mathrm{rd} 1$ photoreceptors exhibited a clear center-to-periphery negative gradient (data not shown), which conforms to the progression of $\mathrm{rd} 1$ retinal degeneration (Carter-Dawson et al., 1978). In addition to the ONL labeling there was an occasional staining of structures in the inner nuclear layer (INL), but this occurred in both genotypes.

The PAR antibody staining suggested increased poly(ADP)ribosylation in $\mathrm{rd} 1$ photoreceptors. To assess the temporal dynamics of the ONL poly(ADP)-ribosylation during the degeneration, we analyzed the proportional extent of PAR staining in retinas from $\mathrm{P} 7$ to $\mathrm{P} 15$, which corresponds to the main degeneration period of the rd1 model (Chang et al., 1993; PorteraCailliau et al., 1994). Retinas from wt mice were essentially devoid of PAR-positive ONL cells at any time point, whereas PAR staining appeared in $\mathrm{rd} 1 \mathrm{ONL}$ at P9, which correlates well with the onset of rd1 degeneration (Chang et al., 1993; Portera-Cailliau et 
al., 1994), and remained elevated throughout the studied period (Fig. 2G).

Second, we studied PARP activity directly using an in situ assay. PARP activity in rd1 ONL nuclei was only slightly higher than in wt at P7 and P9, but was profoundly increased for the remainder of the studied period (Fig. 2G). Similar to the PAR staining, the PARP activity in the P11 rd1 retinas showed a clear center-toperiphery gradient, which is in accordance with the finding that PARP activity staining showed extensive overlap with PAR immunofluorescence staining (data not shown). Moreover, both rd1 and wt retinas occasionally showed positive cells in the INL at P11 (Fig. 3; for views of full retinal cross sections, refer to supplemental Figure 3, available at www.jneurosci. org as supplemental material), just as was seen with the PAR staining, suggesting high levels of PARP activity in certain inner retina neurons at this stage. It is likely that this activity is related to the residual developmental INL apoptosis at P11 (Young, 1984), because an involvement of PARP-1 in apoptosis in the developing nervous system has recently been inferred (Sugo et al., 2007). It should be noted that in the strict sense of the word, the in situ assay does not detect PARP activity but activated PARP. The discrepancy between the numbers of PAR-positive cells and PARP activity-positive cells (Fig. 2G) may relate to PARP being activated but prevented from being active under in vivo conditions, for instance by a scarcity of substrates.

\section{Correlation of PARP activity with cell} death markers

The temporal profile of the rd1 PARP activity (above) suggested a relationship with $\mathrm{rd} 1$ photoreceptor death. We therefore performed TUNEL assays to determine the amount of dying cells in the retina at the same time points. The temporal appearance of TUNEL-positive cells in the rd1 ONL showed a striking correlation with the PARP activity (Fig. $2 G)$. In addition, when staining for PAR or PARP activity was combined with the TUNEL assay on P11 rd1 retinal sections, a high degree of overlap was observed. Of the PARP activity-positive cells, $87.8 \pm 2.1 \%($ mean \pm SEM; $n=6)$ were also TUNEL positive (Fig. $3 F$ ). Similarly, $88.9 \pm 2.4 \%(n=6)$ of PAR immunofluorescent cells were also TUNEL positive (colabeling for in vivo samples not shown, but see Fig. $4 E, F$ for the in vitro situation).

We showed previously that, when used in high concentrations, fluorescent avidin can be efficiently used to detect oxidatively damaged DNA in rd1 photoreceptors and, as such, labels

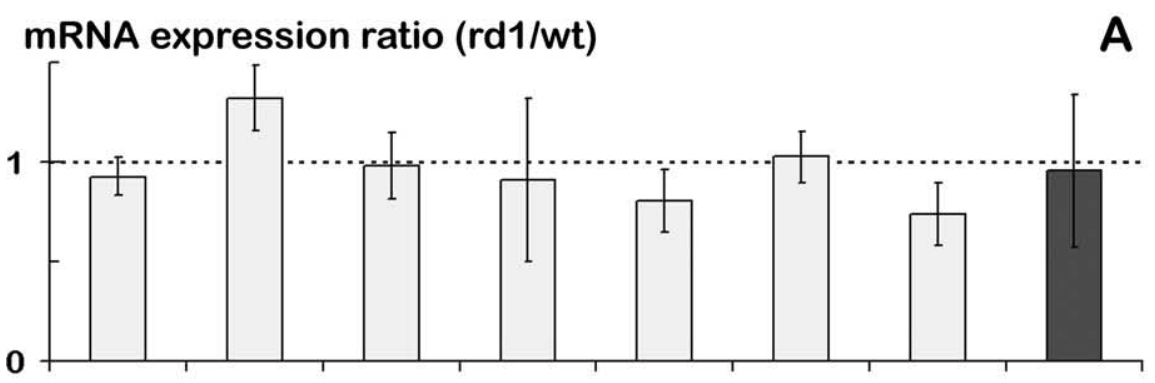

PARP1 PARP2 PARP6 PARP7 PARP8 PARP14 PARP16 PARG
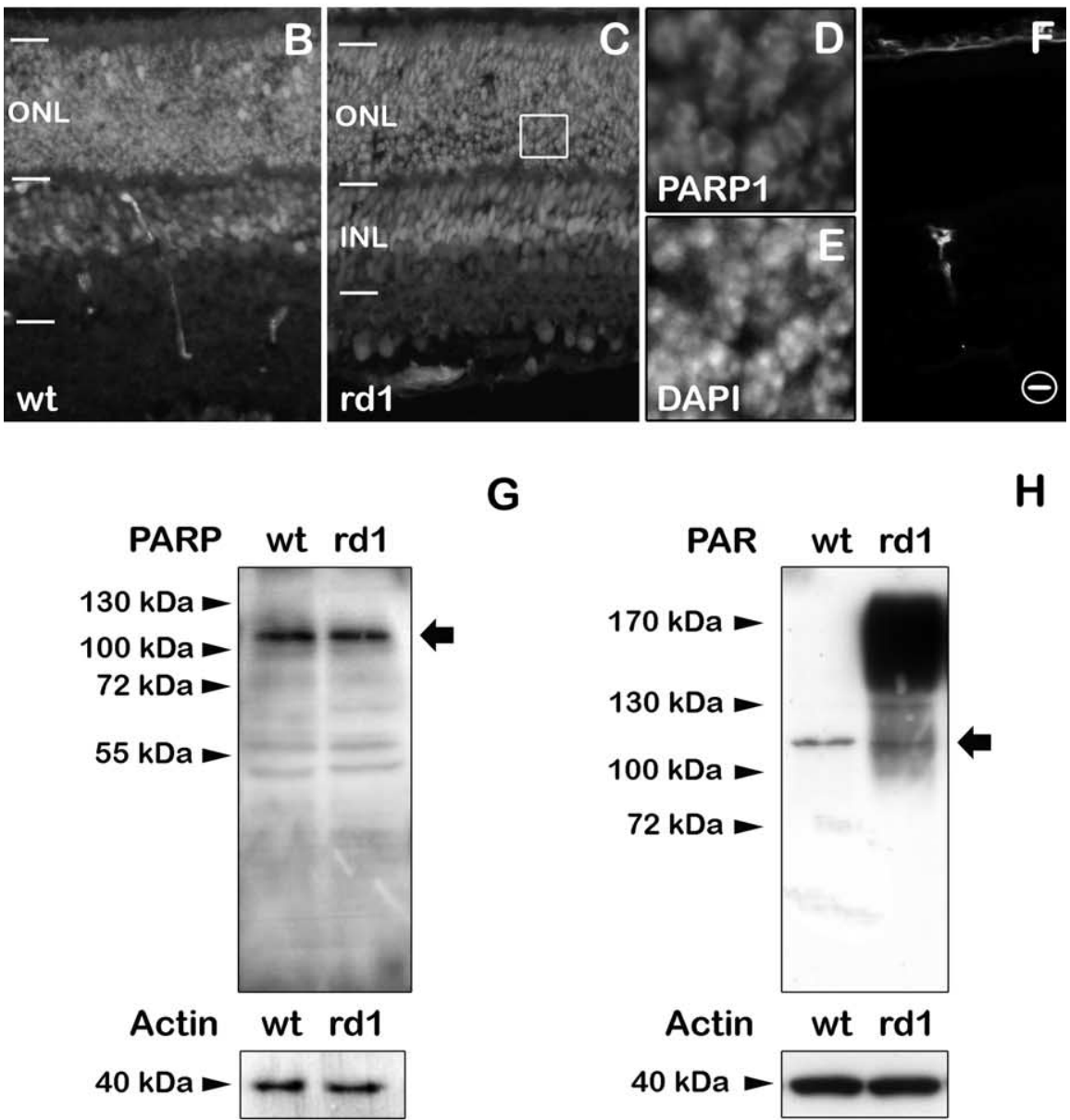

G

Figure 1. Expression of PARP in rd1 and wt retina. The mRNA expression ratios of several different PARP isoforms as well as PARG in the rd1 retina were analyzed by microarray techniques and found to be unchanged when compared with corresponding wt retina (number of independent sample pairs, 3-5; error bars indicate SEM) (A). Immunofluorescent staining for PARP-1 showed its expression in all parts of the retina, including the $0 \mathrm{NL}$, but did not reveal important differences in expression or localization of PARP between wt and $\mathrm{rd}(\boldsymbol{B}, \boldsymbol{C})$. The strong staining in the INL relates to residual endogenous mouse Ig $\mathrm{G}$ in retinal blood vessels, which is recognized by the fluorescent secondary anti-mouse lgG antibody [see also negative control $(-)(\boldsymbol{F})$ ]. PARP-1 immunofluorescence was mostly located in photoreceptor nuclei as evidenced by colabeling with 4',6-diamidino-2phenylindole (DAPI) ( $\boldsymbol{D}, \boldsymbol{E}$; magnification of area labeled with rectangle in $\boldsymbol{C}$. Immunoblotting for PARP-1 also demonstrated equal protein expression levels in wt and $\mathrm{rd} 1 \mathrm{retina}(\boldsymbol{G})$. Immunoblotting for the PARP product PAR showed one band at $\sim 116 \mathrm{kDa}$ (black arrow), which most likely represented PARP itself. In striking contrast to the other findings, PAR immunoblot showed a strong labeling in rd1 samples of proteins ranging from 140 to $240 \mathrm{kDa}$, suggesting rd1-dependent poly(ADP)-ribosylation $(\boldsymbol{H})$. The black arrowheads in $\mathbf{G}$ and $\boldsymbol{H}$ indicate positions of molecular weight standards; actin immunolabeling was used as loading control.

the same cells as antibodies to 8 -OHdG (8-oxoguanine) (Miranda-Sanz et al., 2007). The structure of the latter molecule is similar to that of biotin (i.e., the conventional ligand for avidin). To try to relate excessive PARP activation to its main activator, DNA damage, we combined PAR immunofluorescence with avidin staining at P11. The avidin staining demonstrated 

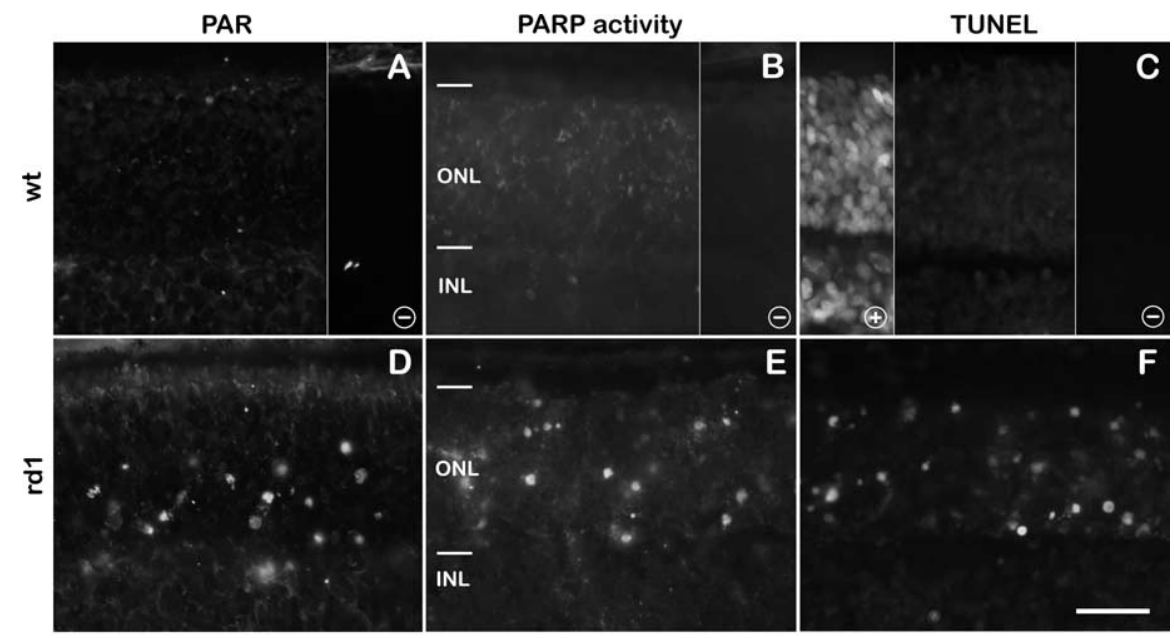

$\%$ positive cells

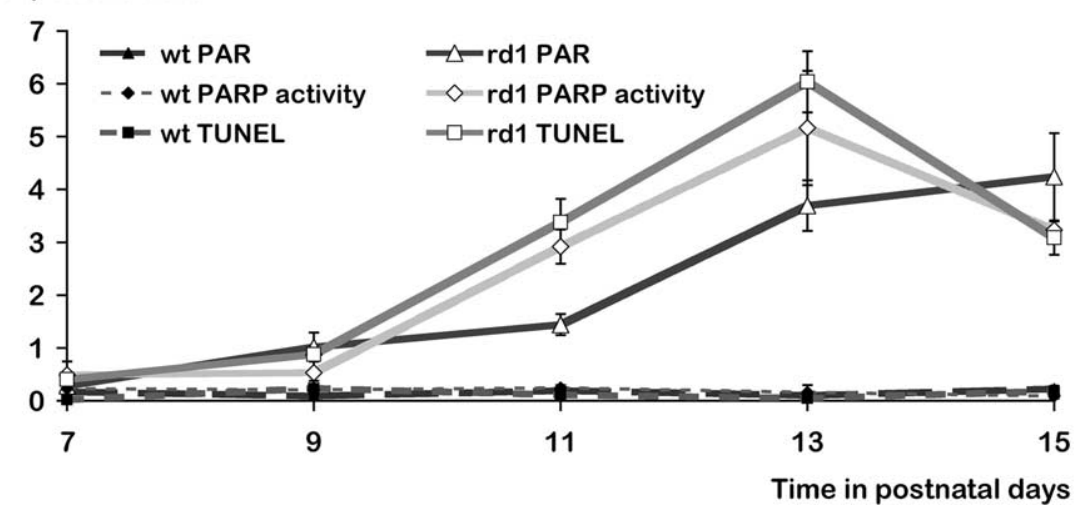

Figure 2. Biochemical markers for PARP activity and cell death are increased in the rd1 retina. PAR immunofluorescence $(\boldsymbol{A}, \boldsymbol{D})$, PARP in situ activity assay $(\boldsymbol{B}, \boldsymbol{E})$, and TUNEL staining for dying cells $(\boldsymbol{C}, \boldsymbol{F})$ were used to assess the extent of PARP activity and cell death in wt $(\boldsymbol{A}-\boldsymbol{C})$ and rd1 retina $(\boldsymbol{D}-\boldsymbol{F})$ at P11. All three markers showed an increase in the percentage of positive cells in the rd1 ONL. Scale bar, $50 \mu \mathrm{m}$. The graph in $\mathbf{G}$ shows the development of these markers in wt and $\mathrm{rd} 1 \mathrm{ONL}$ during the second postnatal week. Note that PARP activity and TUNEL positivity were strongly increased in rd1 retina from P11 onward and generally followed the same pattern. When compared with these markers, PAR immunoreactivity was also increased in the rd1 retina but slightly delayed. Negative $(-)$ and positive $(+)$ controls for PAR, PARP activity, and TUNEL are shown as split pictures in $\boldsymbol{A}-\boldsymbol{C}$. Some of the INL labeling in $\boldsymbol{A}$ and $\boldsymbol{D}$ relates to $\lg G$ in blood vessels. The graph shows the mean \pm SEM for three to eight animals for each genotype.

extensive overlap with the PAR staining in the rd1 ONL, with $75.9 \pm 4.2 \%(n=6)$ of PAR-positive cells also being avidin positive (Fig. $3 P-R$ ). This finding suggests that excessive PARP activity is caused by or otherwise related to oxidatively damaged DNA.

Mitochondrial release of apoptosis-inducing factor (AIF) and translocation to the nucleus has been shown to be dependent on PARP activity, as well as being an essential step toward PARPmediated cell death (Yu et al., 2002). To address a possible relationship between these two factors, AIF immunofluorescence was colabeled with PARP activity. Nuclear AIF staining at P11 was significantly increased in the $\mathrm{rd} 1 \mathrm{ONL}$ when compared with its wt counterpart ( $\mathrm{rd1}, 5.1 \pm 0.4 \%$; wt, $2.0 \pm 0.7 \%$; $n=6$; $p<$ 0.05), confirming previous results from Sanges et al. (2006). Furthermore, in a subset of the rd1 ONL cells, PARP activity and nuclear AIF coincided (Fig. 3J-L), suggesting a connection between PARP activity and AIF nuclear translocation.

\section{PARP inhibition protects rd1 photoreceptors in vitro}

The importance of PARP activity for the rd1 degeneration was addressed by an in vitro retinal explant system, by which we tested
G

the effect of the highly specific PARP inhibitor PJ34 (Garcia Soriano et al., 2001; Jagtap et al., 2002). Two different culturing regimens were used and are referred to as either short-term or long-term.

To find out whether PJ34 treatment really prevented the formation of poly(ADP)-ribosylated proteins, shortterm in vitro retinal cultures starting at $\mathrm{P} 5$ were treated from P7 onward, ended at either P9 or P11, and subsequently subjected to Western blot for PAR. The PAR Western blot in general showed a clear reduction in the amount of poly(ADP)ribosylated proteins in the treated specimens at both time points (P9: $50.3 \pm$ $2.1 \%, n=2$; P11: $53.2 \pm 25.2 \%, n=3$ ) (Fig. 4), confirming the efficacy of the inhibitory treatment. The main poly(ADP)ribosylated band at $116 \mathrm{kDa}$ corresponding most likely to PARP itself was more prominent at P11 (100\%) than at P9 (92 $13.6 \%$; $n=2)$, indicating that PARP activity had increased in this time frame. This is in accordance with the in vivo data presented in Figure 2. High molecular weight poly(ADP)-ribosylated bands appear to be more abundant at P9 than at P11. In the untreated specimens, the blots show a group of additional poly(ADP)ribosylated bands at relatively low molecular weights between 33 and $45 \mathrm{kDa}$. Interestingly, we previously demonstrated a strong increase in the activity of calpains in $\mathrm{rd} 1$ photoreceptor degeneration (Paquet-Durand et al., 2006), and the low molecular weight poly(ADP)-ribosylated bands are compatible with products of calpain cleavage of PARP (Buki et al., 1997).

Short-term cultures from P5 to P11 also showed a significant reduction in the number of PAR-positive rd1 ONL cells in treated specimens (PJ34, $0.4 \pm 0.1 \%$; vehicle, $1.1 \pm 0.2 \% ; n=6$; $p<0.05$ ) (Fig. 5C,D), again confirming that PJ34 correlated with a reduction of PARP activity. Importantly, the treatment with PJ34 also led to a significant reduction in the number of TUNELpositive ONL cells (PJ34, $3.7 \pm 0.4 \%$; vehicle, $4.6 \pm 0.5 \% ; n=6$; $p<0.05$ ) (Fig. 5E,F), indicating that reduced PARP activity helps in preventing photoreceptor cell death. The decrease in PAR staining was proportionally larger than the decrease in TUNEL staining. This might be attributed to a difference in sensitivities between PAR and TUNEL stainings but could also be attributable to other cell death mechanisms becoming activated when PARP is inhibited. We also performed the PARP activity assay on these preparations but were unable to detect any difference between treated and untreated retinas in this respect (data not shown). However, PARP inhibition by PJ34 can be reversed (Jagtap et al., 2002). PARP activity in the unfixed sections of the PJ34-treated retinas could thus be expected to be resumed during the assay, in which for obvious reasons no inhibitor is included, because this would block any enzymatic activity (see Materials and Methods). The observed in situ PARP activity in the treated 
retinas therefore indicates that the upstream events that activated PARP were not affected by the drug.

To ascertain that the decreased number of TUNEL-positive cells observed in the short-term cultures translated into an increased survival of photoreceptors, we used a long-term treatment in which the retinal explants were cultured from P5 until P25. This culture period covers in principle the complete rdl rod degeneration, so that by the end only one to two rows of photoreceptors (mostly cones) remain in the $\mathrm{rd} 1 \mathrm{ex}$ plants (Ahuja et al., 2005). Long-term PJ34 treatment significantly increased the number of surviving $\mathrm{rd} 1$ photoreceptor rows by $\sim 40 \%$ (PJ34, $2.4 \pm 0.0$ rows; vehicle, $1.7 \pm$ 0.0 rows; $n=6 ; p<0.001$ ) (Fig. 6). This finding is thus in line with the lower number of dying cells observed in the corresponding short-term cultures. Together, these results show that PJ34 treatment led to a reduction of both PARP activity and rd1 photoreceptor cell death, proposing a causal connection between the two.

\section{Discussion}

We here show that inherited photoreceptor cell death in the rd1 mouse involves PARP enzymatic activity, which is novel information. PARP activity per se as well as its product, PAR, were unequivocally and dramatically increased in the ONL of the rd1 retina, where it correlated with the temporal characteristics of the rd1 degeneration (CarterDawson et al., 1978; Chang et al., 1993; Portera-Cailliau et al., 1994). Furthermore, both PARP activity and product displayed a striking colocalization with dying, TUNELpositive photoreceptors, indicating an involvement of excessive PARP activity in $\mathrm{rd} 1$ rod cell death. In line with this, inhibition of PARP activity reduced the number of PARand TUNEL-positive ONL cells in shortterm cultures, an effect that in long-term cultures was translated to an increased number of surviving photoreceptors.

Gene transcription and protein expression of PARP were unchanged when rd 1 and wt retinas were compared, and we did not observe any differential tissue localization of PARP protein between the genotypes. The excessive PARP activity in the dying rd1 photoreceptors must therefore to a large extent stem from activation of already existing PARP molecules. The level of PAR in a cell results from the balance between PARP and PARG activity. Its accumulation in photoreceptor cells could thus in principle be attributable to either increased PARP activity or decreased PARG activity or a combination of both. Because PAR correlates and colocalizes with increased PARP activity in photoreceptor cells, the first possibility seems more likely.
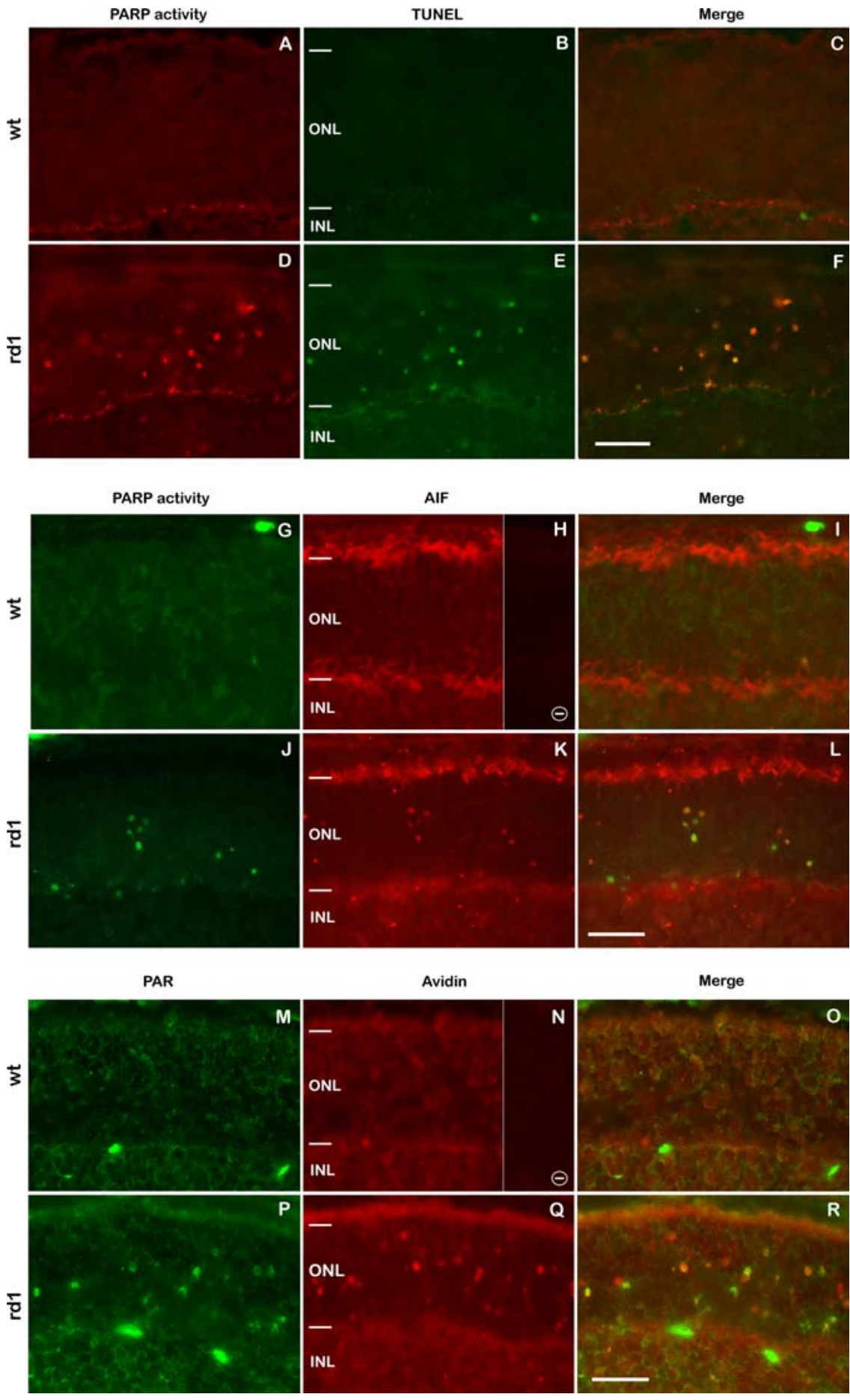

Figure 3. PARP activity and cell death. The number of PARP activity-positive cells in the rd1 ONL (D) was strongly increased when compared with wt $(\boldsymbol{A})$. The in situ PARP activity colocalized to a large extent with TUNEL staining for dying cells $(\boldsymbol{B}, \boldsymbol{C}, \boldsymbol{E}, \boldsymbol{F})$. Immunofluorescence for AIF strongly labeled the photoreceptor segments and outer plexiform layer, areas which are known to host large numbers of mitochondria. Nuclear translocation of AlF was not observed in wt $(\boldsymbol{H})$ but in a subset of rd1 photoreceptors $(\boldsymbol{K})$ where it overlapped with PARP activity staining $(J$; merged image in $L)$. Similarly, avidin staining for oxidatively damaged DNA did not show appreciable amounts of positive cells in the wt $(\boldsymbol{N})$ but revealed a large number of positive cells in the rd1 ONL (Q). PAR immunofluorescent staining $(\boldsymbol{M}, \boldsymbol{P}$; merged with avidin in $\mathbf{O}, \boldsymbol{R})$ was again morefrequent in rd 1 photoreceptors, where it overlapped to a large extent with avidin $(\boldsymbol{R})$, suggesting that PARP in these cells was activated by DNA damage. The stainings are representative of at least six specimens. Some of the INL labeling in $\boldsymbol{M}, \boldsymbol{P}, \mathbf{O}$, and $\boldsymbol{R}$ relates to $\lg \mathrm{G}$ in blood vessels. Scale bars, $50 \mu \mathrm{m}$.

These findings underline the necessity to extend analyses from mRNA and protein to the actual catalytic, enzymatic level, preferably with cellular resolution. We have in this context similarly established that CaMKII (calmodulin kinase II) as well as calpain 

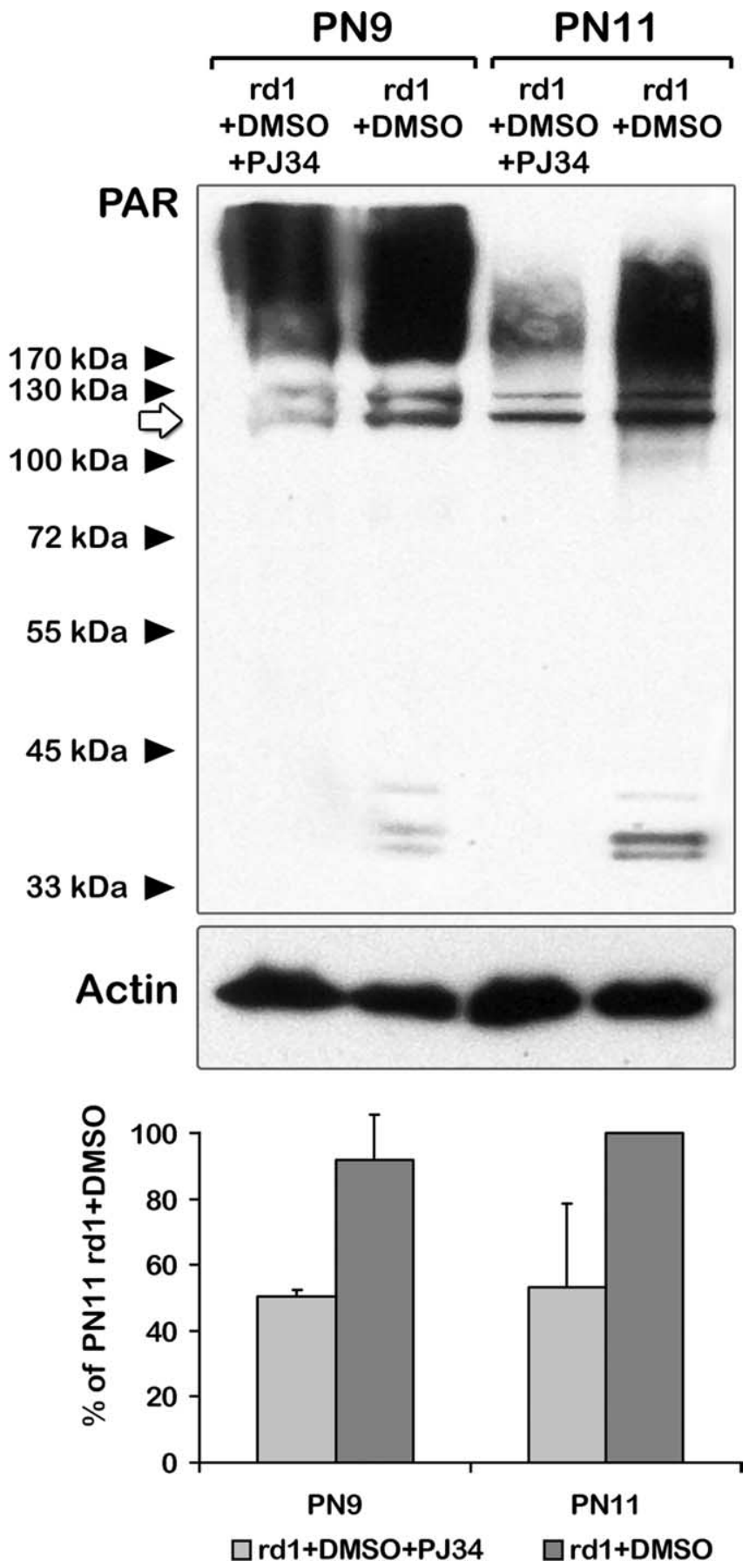

Figure 4. PARP inhibition decreases the amount of poly(ADP)-ribosylated proteins in rd1 short-term retinal cultures. Short-term rd1 retinal cultures (starting at $P 5$ ) were treated with the PARP inhibitor PJ34 from P7 to either P9 or P11. Immunoblot for poly(ADP)-ribosylated proteins shows a clear reduction in the PJ34-treated specimens compared with untreated (DMSO only) control. The band at $116 \mathrm{kDa}$ (white arrow) most likely represents PARP itself and was considered for the quantification of treatment effects. The Western blots are representative for two to three individual retinas from different animals, respectively; the black arrowheads indicate positions of molecular weight standards; actin immunolabeling was used as loading control; bar graphs show the mean \pm SEM.

type proteases are strongly activated in $\mathrm{rd} 1$ photoreceptors, whereas their expression levels remain unchanged (Hauck et al., 2006; Paquet-Durand et al., 2006, 2007).

PARP is activated by DNA strand breaks and helps in their subsequent repair (Schreiber et al., 2006). Because it is generally

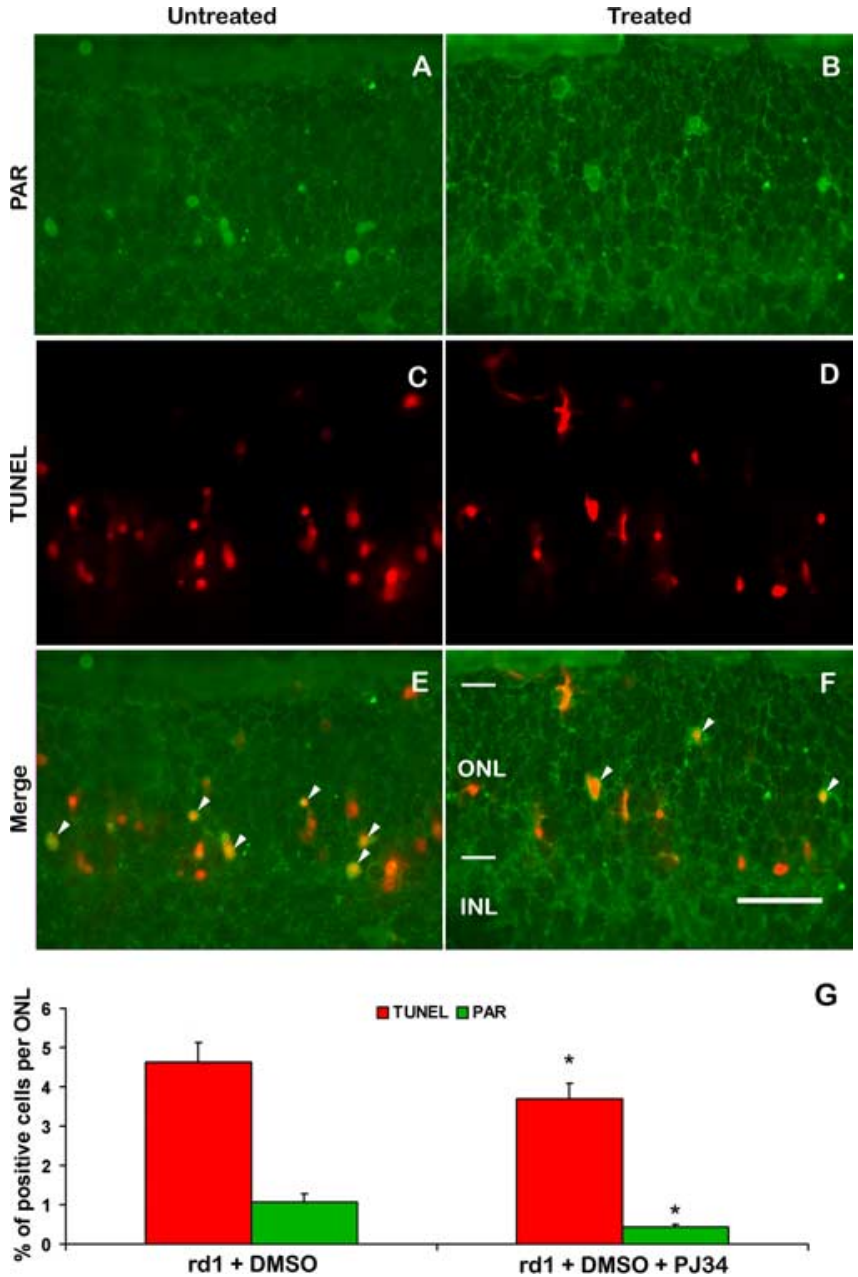

Figure 5. PARP inhibition decreases the number of dying rd1 photoreceptors in short-term retinal cultures. In short-term cultures (P5-P11) both the number of PAR-immunofluorescent cells $(\boldsymbol{A}, \boldsymbol{B}, \boldsymbol{G})$ and the number of TUNEL-positive cells $(\boldsymbol{C}, \boldsymbol{D}, \boldsymbol{G})$ in the rd1 $0 \mathrm{NL}$ were significantly decreased by PJ34 treatment, suggesting a protective effect of the PARP inhibitor. The merged pictures $(\boldsymbol{E}, \boldsymbol{F})$ show a high degree of overlap between PAR immunofluorescence and TUNEL staining, indicating that excessive PARP activity may cause cell death. The white arrowheads indicate some colabeled cells. The stainings are representative of six specimens; bar graphs show the mean \pm SEM; $n=6$ animals; ${ }^{*} p<0.05$. Scale bar, $50 \mu \mathrm{m}$.

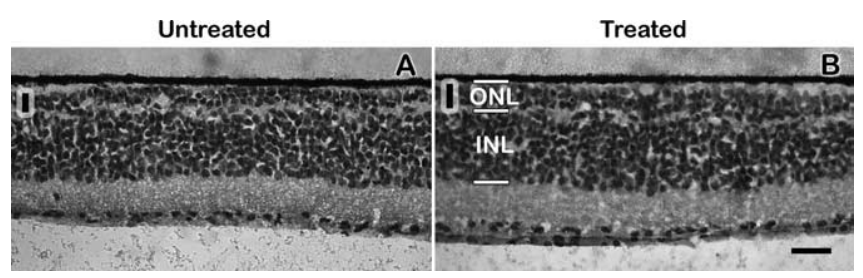

Figure 6. PARP inhibition partially rescues $r d 1$ photoreceptors in long-term retinal cultures. Long-term treatment with PJ34 (P5-P25) (A) resulted in a significant increase in the number of surviving photoreceptors when compared with untreated specimens $(\boldsymbol{B})$. The highlighted, upright black bars in $\boldsymbol{A}$ and $\boldsymbol{B}$ indicate the thickness of the ONL. The stainings are representative of six specimens. Scale bar, $50 \mu \mathrm{m}$.

agreed that DNA is continually damaged and restored throughout the life of most cells (Lindahl and Wood, 1999), we may assume a certain basal level of PARP activity in all cells, at all times. However, at each time point studied, the in situ PARP activity assay identified a subpopulation of rd1 photoreceptor cells with very high PARP activation, well beyond any level displayed by the surrounding cells. 
Moreover, we were able to correlate such overactivated PARP with oxidatively damaged DNA, which we previously linked with the death of the rd1 photoreceptors (Miranda-Sanz et al., 2007). It therefore seems likely that the excessive PARP activity results from increased DNA damage of the rd1 rods, which together contributes to the catastrophic events that kill these cells. This scheme implies a relatively late onset of the pathological PARP activity, occurring when perhaps other aspects of cellular metabolism have already become compromised, and may therefore explain the absence of complete protection by the PJ34-based PARP inhibition. However, we cannot at this stage exclude the possibility that the protective effects of PARP inhibition are circumvented by alternative death pathways that become activated when PARP is unavailable.

AIF has recently been shown to translocate from mitochondria to nuclei in degenerating $\mathrm{rd} 1$ photoreceptor cells (Sanges et al., 2006). Nuclear translocation of AIF is known to lead to activation of endonucleases, widespread DNA fragmentation, and subsequent cell death (Krantic et al., 2007). Intriguingly, AIF has also been suggested to act as a mediator of PARP-induced apoptosis in cultured neurons and other cells (Yu et al., 2002; Cipriani et al., 2005; Culmsee et al., 2005; Yu et al., 2006). We think that the data presented here connect these findings, because PARP activity was observed to be involved in the rd1 degeneration as well as to correspond with AIF translocation. We thus propose that overactivated PARP is a trigger for AIF-mediated death of rd1 rod photoreceptors. Whether such deleterious effects of excess PARP activity are conveyed by its consumption of $\mathrm{NAD}^{+}$(Sims et al., 1983; Du et al., 2003), by production of poly(ADP)-ribosylated proteins (Yu et al., 2006), such as those observed in our Western blots, or by free PAR polymers, as seen in the PARP-dependent cell death of primary cortical neurons (Andrabi et al., 2006), remains to be studied.

In summary, our results provide new insights into the mechanisms of retinal photoreceptor degeneration. This could have a bearing on our understanding of neurodegeneration overall, and also suggests that the therapeutical possibilities of PARP inhibition should include retinal diseases like RP.

\section{References}

Ahuja P, Caffe AR, Ahuja S, Ekström P, van Veen T (2005) Decreased glutathione transferase levels in $\mathrm{rd} 1 / \mathrm{rd} 1$ mouse retina: replenishment protects photoreceptors in retinal explants. Neuroscience 131:935-943.

Andrabi SA, Kim NS, Yu SW, Wang H, Koh DW, Sasaki M, Klaus JA, Otsuka T, Zhang Z, Koehler RC, Hurn PD, Poirier GG, Dawson VL, Dawson TM (2006) Poly(ADP-ribose) (PAR) polymer is a death signal. Proc Natl Acad Sci USA 103:18308-18313.

Azadi S, Paquet-Durand F, Medstrand P, van Veen T, Ekström P (2006) Up-regulation and increased phosphorylation of protein kinase $\mathrm{C}$ (PKC) $\delta, \mu$ and $\theta$ in the degenerating rd1 mouse retina. Mol Cell Neurosci 31:759-773.

Azadi S, Johnson L, Paquet-Durand F, Perez MTR, Zhang Y, Ekström P, van Veen T (2007) CNTF+BDNF treatment and neuroprotective pathways in the rd1 mouse retina. Brain Res 1129:116-129.

Bakondi E, Bai P, Szabo EE, Hunyadi J, Gergely P, Szabo C, Virag L (2002) Detection of poly(ADP-ribose) polymerase activation in oxidatively stressed cells and tissues using biotinylated NAD substrate. J Histochem Cytochem 50:91-98.

Bonicalzi ME, Haince JF, Droit A, Poirier GG (2005) Regulation of poly(ADP-ribose) metabolism by poly(ADP-ribose) glycohydrolase: where and when? Cell Mol Life Sci 62:739-750.

Bowes C, Li T, Danciger M, Baxter LC, Applebury ML, Farber DB (1990) Retinal degeneration in the rd mouse is caused by a defect in the beta subunit of rod cGMP-phosphodiesterase. Nature 347:677-680.

Buki KG, Bauer PI, Kun E (1997) Isolation and identification of a proteinase from calf thymus that cleaves poly(ADP-ribose) polymerase and histone H1. Biochim Biophys Acta 1338:100-106.
Carter-Dawson LD, LaVail MM, Sidman RL (1978) Differential effect of the rd mutation on rods and cones in the mouse retina. Invest Ophthalmol Vis Sci 17:489-498.

Chang GQ, Hao Y, Wong F (1993) Apoptosis: final common pathway of photoreceptor death in rd, rds, and rhodopsin mutant mice. Neuron 11:595-605.

Chiang SK, Lam TT (2000) Post-treatment at 12 or 18 hours with 3-aminobenzamide ameliorates retinal ischemia-reperfusion damage. Invest Ophthalmol Vis Sci 41:3210-3214.

Cipriani G, Rapizzi E, Vannacci A, Rizzuto R, Moroni F, Chiarugi A (2005) Nuclear poly(ADP-ribose) polymerase-1 rapidly triggers mitochondrial dysfunction. J Biol Chem 280:17227-17234.

Cobbs WH, Pugh EN (1985) Cyclic GMP can increase rod outer-segment light sensitive current 10-fold without delay of excitation. Nature 313:585-587.

Culmsee C, Zhu C, Landshamer S, Becattini B, Wagner E, Pellecchia M, Blomgren K, Plesnila N (2005) Apoptosis-inducing factor triggered by poly(ADP-ribose) polymerase and Bid mediates neuronal cell death after oxygen-glucose deprivation and focal cerebral ischemia. J Neurosci 25:10262-10272.

Delyfer MN, Leveillard T, Mohand-Said S, Hicks D, Picaud S, Sahel JA (2004) Inherited retinal degenerations: therapeutic prospects. Biol Cell 96:261-269.

Doonan F, Donovan M, Cotter TG (2003) Caspase-independent photoreceptor apoptosis in mouse models of retinal degeneration. J Neurosci 23:5723-5731.

Du L, Zhang X, Han YY, Burke NA, Kochanek PM, Watkins SC, Graham SH, Carcillo JA, Szabo C, Clark RS (2003) Intra-mitochondrial poly(ADPribosylation) contributes to NAD + depletion and cell death induced by oxidative stress. J Biol Chem 278:18426-18433.

Farber DB, Lolley RN (1974) Cyclic guanosine monophosphate: elevation in degenerating photoreceptor cells of the $\mathrm{C} 3 \mathrm{H}$ mouse retina. Science 186:449-451.

Fossati S, Cipriani G, Moroni F, Chiarugi A (2007) Neither energy collapse nor transcription underlie in vitro neurotoxicity of poly(ADP-ribose) polymerase hyper-activation. Neurochem Int 50:203-210.

Fox DA, Poblenz AT, He L (1999) Calcium overload triggers photoreceptor apoptotic cell death in chemical-induced and inherited retinal degenerations. Ann NY Acad Sci 893:282-285.

Garcia Soriano F, Virag L, Jagtap P, Szabo E, Mabley JG, Liaudet L, Marton A, Hoyt DG, Murthy KG, Salzman AL, Southan GJ, Szabo C (2001) Diabetic endothelial dysfunction: the role of poly(ADP-ribose) polymerase activation. Nat Med 7:108-113.

Gavrieli Y, Sherman Y, Ben-Sasson SA (1992) Identification of programmed cell death in situ via specific labeling of nuclear DNA fragmentation. J Cell Biol 119:493-501.

Goebel DJ, Winkler BS (2006) Blockade of PARP activity attenuates poly(ADP ribosyl)ation but offers only partial neuroprotection against NMDA-induced cell death in the rat retina. J Neurochem 98:1732-1745.

Hauck SM, Ekström PA, Ahuja-Jensen P, Suppmann S, Paquet-Durand F, van Veen T, Ueffing M (2006) Differential modification of phosducin protein in degenerating $\mathrm{rd} 1 \mathrm{retina}$ is associated with constitutively active $\mathrm{Ca}^{2+} /$ calmodulin kinase II in rod outer segments. Mol Cell Proteomics $5: 324-336$.

Herse P (2005) Retinitis pigmentosa: visual function and multidisciplinary management. Clin Exp Optom 88:335-350.

Krantic S, Mechawar N, Reix S, Quirion R (2007) Apoptosis-inducing factor: a matter of neuron life and death. Prog Neurobiol 81:179-196.

Jagtap P, Szabo C (2005) Poly (ADP-ribose) polymerase and the therapeutic effects of its inhibitors. Nat Rev Drug Discov 4:421-440.

Jagtap P, Soriano FG, Virag L, Liaudet L, Mabley J, Szabo E, Hasko G, Marton A, Lorigados CB, Gallyas Jr F, Sumegi B, Hoyt DG, Baloglu E, VanDuzer J, Salzman AL, Southan GJ, Szabo C (2002) Novel phenanthridinone inhibitors of poly (adenosine $5^{\prime}$-diphosphate-ribose) synthetase: potent cytoprotective and antishock agents. Crit Care Med 30:1071-1082.

Kim DH, Kim JA, Choi JS, Joo CK (2002) Activation of caspase-3 during degeneration of the outer nuclear layer in the rd mouse retina. Ophthalmic Res 34:150-157.

Koh DW, Dawson TM, Dawson VL (2005) Mediation of cell death by poly(ADP-ribose) polymerase-1. Pharmacol Res 52:5-14.

Lindahl T, Wood RD (1999) Quality control by DNA repair. Science 286:1897-1905. 
McLaughlin ME, Ehrhart TL, Berson EL, Dryja TP (1995) Mutation spectrum of the gene encoding the $\beta$ subunit of rod phosphodiesterase among patients with autosomal recessive retinitis pigmentosa. Proc Natl Acad Sci USA 92:3249-3253.

Mendoza-Alvarez H, Alvarez-Gonzalez R (1993) Poly(ADP-ribose) polymerase is a catalytic dimer and the automodification reaction is intermolecular. J Biol Chem 268:22575-22580.

Miki K, Uehara N, Shikata N, Matsumura M, Tsubura A (2007) Poly (ADPribose) polymerase inhibitor 3-aminobenzamide rescues $N$-methyl- $N$ nitrosourea-induced photoreceptor cell apoptosis in Sprague-Dawley rats through preservation of nuclear factor-kappaB activity. Exp Eye Res 84:285-292.

Miranda-Sanz M, Johnson LE, Ahuja S, Ekström PA, Romero J, van Veen T (2007) Significant photoreceptor rescue by treatment with a combination of antioxidants in an animal model for retinal degeneration. Neuroscience 145:1120-1129.

Paquet-Durand F, Azadi S, Hauck SM, Ueffing M, van Veen T, Ekström P (2006) Calpain is activated in degenerating photoreceptors in the rd1 mouse. J Neurochem 96:802-814.

Paquet-Durand F, Johnson L, Ekström P (2007) Calpain activity in retinal degeneration. J Neurosci Res 85:693-702.

Portera-Cailliau C, Sung CH, Nathans J, Adler R (1994) Apoptotic photoreceptor cell death in mouse models of retinitis pigmentosa. Proc Natl Acad Sci USA 91:974-978.

Sanges D, Comitato A, Tammaro R, Marigo V (2006) Apoptosis in retinal degeneration involves cross-talk between apoptosis-inducing factor (AIF) and caspase-12 and is blocked by calpain inhibitors. Proc Natl Acad Sci USA 103:17366-17371.
Schreiber V, Dantzer F, Ame JC, de Murcia G (2006) Poly(ADP-ribose): novel functions for an old molecule. Nat Rev Mol Cell Biol 7:517-528.

Scott GS, Szabo C, Hooper DC (2004) Poly(ADP-ribose) polymerase activity contributes to peroxynitrite-induced spinal cord neuronal cell death in vitro. J Neurotrauma 21:1255-1263.

Sims JL, Berger SJ, Berger NA (1983) Poly(ADP-ribose) polymerase inhibitors preserve nicotinamide adenine dinucleotide and adenosine $5^{\prime}$ triphosphate pools in DNA-damaged cells: mechanism of stimulation of unscheduled DNA synthesis. Biochemistry 22:5188-5194.

Sugo N, Niimi N, Aratani Y, Masutani M, Suzuki H, Koyama H (2007) Decreased PARP-1 levels accelerate embryonic lethality but attenuate neuronal apoptosis in DNA polymerase beta-deficient mice. Biochem Biophys Res Commun 354:656-661.

Yoshizawa K, Kiuchi K, Nambu H, Yang J, Senzaki H, Kiyozuka Y, Shikata N, Tsubura A (2002) Caspase-3 inhibitor transiently delays inherited retinal degeneration in $\mathrm{C} 3 \mathrm{H}$ mice carrying the rd gene. Graefes Arch Clin Exp Ophthalmol 240:214-219.

Young RW (1984) Cell death during differentiation of the retina in the mouse. J Comp Neurol 229:362-373.

Yu SW, Wang H, Poitras MF, Coombs C, Bowers WJ, Federoff HJ, Poirier GG, Dawson TM, Dawson VL (2002) Mediation of poly(ADP-ribose) polymerase-1-dependent cell death by apoptosis-inducing factor. Science 297:259-263.

Yu SW, Andrabi SA, Wang H, Kim NS, Poirier GG, Dawson TM, Dawson VL (2006) Apoptosis-inducing factor mediates poly(ADP-ribose) (PAR) polymer-induced cell death. Proc Natl Acad Sci USA 103:18314-18319.

Zeiss CJ, Neal J, Johnson EA (2004) Caspase-3 in postnatal retinal development and degeneration. Invest Ophthalmol Vis Sci 45:964-970. 